\title{
Parent Preferences for Health Outcomes Associated with Autism Spectrum Disorders
}

\author{
Tara A. Lavelle ${ }^{1,2}$ D $\cdot$ Milton C. Weinstein ${ }^{3} \cdot$ Joseph P. Newhouse ${ }^{4,5,6,7} \cdot$ Kerim Munir $^{8,9} \cdot$ Karen A. Kuhlthau $^{9,10}$. \\ Lisa A. Prosser ${ }^{11}$
}

Published online: 21 March 2019

(c) The Author(s) 2019

\begin{abstract}
Background Few studies have used preference-based quality-of-life outcomes to assess how autism spectrum disorders (ASDs) affect children and parents, and none have examined variation by ASD severity.

Objective Our objective was to derive parent valuations of child and parent health associated with varying ASD severity levels.

Methods Parents of children aged 3-17 years with and without ASD were selected from a nationally representative research panel to complete a survey. We asked parents time trade-off (TTO) questions to value their own and their child's current health. Parents of children with ASD were asked to report the severity of their child's core ASD symptoms. We calculated utility values from each TTO amount, and used a two-part regression model to estimate the change in parent-reported child health utility, as well as parent health utility, associated with ASD diagnosis and increasing symptom severity, controlling for respondent and child characteristics.

Results Sixty-nine percent of parents responded (final sample size was 135 in the ASD group and 120 in the comparison group). In adjusted analyses, there was a 0.12 (95\% confidence interval [CI] 0.03-0.21) decrease in the parent-reported health utility of children with ASD, a 15\% decrease from the mean health utility of children without ASD. On average, having a child with ASD was not significantly associated with a decrease in parent health utility, but there was a 0.14 (95\% CI 0.01-0.26) reduction in health utility among parents of children with severe ASD, a 15\% decrease from the comparison group mean. Conclusions Overall, ASD had a significant impact on parent-reported child health utility, and the health utility of parents of children with severe ASD.
\end{abstract}

Electronic supplementary material The online version of this article (https://doi.org/10.1007/s40273-019-00783-8) contains supplementary material, which is available to authorized users.

Tara A. Lavelle

tlavelle@tuftsmedicalcenter.org

1 Center for the Evaluation of Value and Risk in Health, Institute for Clinical Research and Health Policy Studies, Tufts Medical Center, Boston, MA, USA

2 Tufts University School of Medicine, Boston, MA, USA

3 Center for Health Decision Science, Harvard T.H. Chan School of Public Health, Boston, MA, USA

4 Department of Health Care Policy, Harvard Medical School, Boston, MA, USA

5 Department of Health Policy and Management, Harvard T.H. Chan School of Public Health, Boston, MA, USA
6 Harvard Kennedy School, Cambridge, MA, USA

7 National Bureau of Economic Research, Cambridge, MA, USA

8 Division of Developmental Medicine, Boston Children's Hospital, Boston, MA, USA

9 Department of Pediatrics, Harvard Medical School, Boston, MA, USA

10 Division of General Academic Pediatrics, Massachusetts General Hospital, Boston, MA, USA

11 Department of Pediatrics and Communicable Diseases, Child Health Evaluation and Research Center, University of Michigan, Ann Arbor, MI, USA 


\section{Key Points}

Autism spectrum disorder (ASD) has a statistically significant impact on children's health-related quality of life, as measured using parent-reported health utilities.

A child's diagnosis of ASD was, on average, not significantly associated with spillover effects on parent health utility, but there were statistically significant spillover effects among parents of children categorized as most severe.

\section{Introduction}

One in 59 children in the US currently has a diagnosis of autism spectrum disorder (ASD) [1], and there are approximately 52 million individuals with ASD worldwide [2]. As new interventions for ASD are introduced, it is important to evaluate their comparative and cost effectiveness. The quality-adjusted life-year (QALY) is the recommended health outcome measure for cost-effectiveness analyses (CEAs) and weighs time spent in a state of health by the health-related quality of life (HRQOL) or utility value associated with that health state $[3,4]$. Studies typically consider QALYs of the individual patient $[5,6]$, but this framework may be unsuitable for CEAs of ASD interventions. Along with the effect of ASD on an individual's social and behavioral functioning [7], this disorder has large spillover effects on the health and quality of life of family members [8]. For example, parents of children with ASD are at higher risk of depression and anxiety than parents of children without ASD, even those with other developmental disabilities [9-11].

Previous studies have highlighted the importance of including these family spillover effects, which are the secondary impacts of illness on caregivers and other family members, in economic evaluations [12-14]. Spillover effects of pediatric illnesses such as ASD on parents may be especially important to consider as parents serve dual roles as caregivers and healthcare decision makers $[15,16]$. However, only a handful of studies have assessed preferencebased HRQOL outcomes in children with ASD, and the spillover effects on their parents, and none have examined how these health outcomes vary by parent-reported ASD severity [17-22].

The objective of this study is to describe parent-reported health utility values both for children with and without ASD and for their parents. We estimate how a child's diagnosis of ASD is associated with child and parent spillover health utility values, and also describe how these utility values vary across different subtypes and severity levels of ASD.

\section{Methods}

Parents of children with and without ASD were selected from a probability-based US nationally representative research panel (KnowledgePanel; GfK Custom Research, New York, NY, USA) to complete a 25-min online survey in 2011 (a link to the full survey is given in Online Appendix 1A). KnowledgePanel members receive a computer with internet access while participating, or cash incentives equivalent to $\$ 1$ per survey completed for those already with internet access [23]. We conducted focus groups with 21 parents of children with ASD, and cognitive survey pretests with an additional 20 parents, prior to survey administration to aid in question development (details are reported in Online Appendix 1B).

\subsection{Participant Selection}

A two-phase screening process was used to select our two target samples: 1) parents who reported having a child aged 3-17 years diagnosed with ASD (ASD group), and 2) parents who reported having a child aged 3-17 years and no children diagnosed with ASD (comparison group). We defined ASD to include autism, Asperger's syndrome, or pervasive developmental disorder, not otherwise specified (PDD-NOS), consistent with the Diagnostic and Statistical Manual of Mental Disorders, 4th Edition (DSM-IV) diagnostic criteria used at that time. The two-phase screening process used existing information in the KnowledgePanel database to screen respondents based on the age of the children in their household and diagnosis of ASD, and this information was confirmed by parent respondents before the start of the survey (additional details are reported in Online Appendix 1C). After identifying eligible families, we randomly selected one eligible child per household for the parent to reference during the survey (index child). For the ASD group, this was a 3- to 17-year-old child with ASD, and for the comparison group, this was a 3- to 17-year-old child without ASD. We excluded children younger than 3 years of age because ASD is not regularly diagnosed in this group [24].

\subsection{Survey Procedures}

All respondents answered three time trade-off (TTO) questions to value (1) their own current health; (2) their index child's health; and (3) a composite health state that combined the health of both the parent and child (Online Appendix 4). The TTO method estimates each respondent's willingness to trade quantity of life for quality of life [25]. In valuing their own health, we asked parents to report the maximum amount of time he or she would be willing to give up from the end of their life (with a given remaining life 
expectancy of 40 years) to live in a 'perfect' state of health, without any mental, emotional, or physical health problems. To value their child's health, we asked parents to report the maximum amount of time he or she would be willing to give up from the end of their own life for their child to live without any health problems, for the child's remaining life. In a third TTO question, respondents valued a parent and child composite health state by reporting the maximum amount of time they would trade from the end of their own life for both themselves and their child to live their remaining years without health problems. This maximum TTO amount was used for all analyses.

To prepare parents for these three open-ended TTO questions, we first asked parents two dichotomous choice TTO questions before each open-ended question (Online Appendix 1D).

All respondents reported their own and their child's physical and mental health conditions. Parents of children with ASD also reported the severity of their child's core ASD symptoms-restricted interests/repetitive behaviors and social communication problems-on a three-level scale for each, with specific descriptions of each level adapted from DSM, 5th Edition (DSM-V) criteria (Online Appendix 1E) [7].

\subsection{Utility Calculation}

Using the TTO data, we calculated three parent-reported utility values in the ASD and comparison groups: (1) parent health utility; (2) child health utility; and (3) a composite child and parent health utility. We first calculated the disutility of each health state by dividing the maximum amount of time each respondent was willing to trade to avoid each health state by their given life expectancy of 40 years. We then calculated utility values as 1 minus the disutility value (Online Appendix 2).

\subsection{Sample}

We excluded respondents if they traded more time for both the parent-only and the child-only health states compared with the parent/child composite health state (referred to as illogical responses), and also excluded respondents who left all TTO questions blank [26].

\subsection{Unadjusted Analyses}

Using Chi-square tests, we compared respondent and child characteristics between the ASD and comparison groups. We calculated the mean utility values for the ASD and comparison groups and the ASD subgroups. We defined subgroups based on DSM-IV diagnostic categories (autism, Asperger's syndrome, or PDD-NOS) and a three-level ASD composite severity measure (mild, moderate, severe) derived from the parent-reported social communication and behavioral symptom severity questions (Online Appendix 1E). We estimated confidence intervals (CIs) around all mean values using bootstrapping with replacement procedures [27].

\subsection{Adjusted Analyses}

We used a two-part model to evaluate the association between the ASD and ASD subgroups (independent variables) and mean parent-reported utility values for (1) parent health; (2) child health; and (3) composite parent and child health (dependent variables). In the two-part model, the first part used a logistic regression model to estimate the association between ASD and the probability of having a utility value of $<1$, and the second part used a linear regression model to estimate the association between ASD and utility values $<1$. We estimated nine regression equations, with three models for each of the three utility outcomes (parent, child, composite). Predictors for the three models included (1) 'any ASD' versus no ASD; (2) mild, moderate, or severe ASD versus no ASD); and (3) autism, Asperger's, or PDD-NOS versus no ASD. We hypothesized that parents and children with ASD would have lower utility values than the comparison group, and that utility losses would increase with increasing severity of ASD. The parent utility loss associated with ASD is the spillover of the child's ASD on the parent's health utility loss, while the child and parent composite utility loss associated with ASD is a combined estimate of the child's utility loss associated with ASD and the spillover of the child's ASD on the parent's health utility loss.

We controlled for variables that previous research has indicated may have an impact on utility values, independent of health status. These included the respondent's sex, age, race/ethnicity, income, education, marital status, child's sex, age, insurance status, and presence of other children in the household [28-30]. We controlled for the presence (yes/no) of child illnesses not related to the child's ASD diagnosis in all models to minimize differences in health utility that may be associated with other health conditions. We controlled for the presence (yes/no) of parent comorbidities in the models used to estimate child health utility to ensure that parents' health was not impacting their assessment of their child's health. We evaluated the goodness of fit of each linear regression model using the model $R^{2}$, and of each logistic regression model using McFadden's Pseudo $R^{2}$.

\subsection{Sensitivity Analyses}

We reran all adjusted analyses for parent, child, and composite utilities in six sensitivity analyses: (1) tightened our exclusion criteria to further exclude ASD group respondents who did not report that an appropriate provider type (Online 
Appendix 1F) had diagnosed their child, or did not specify a particular subtype of their child's ASD, consistent with DSM-IV diagnostic conventions at the time of the survey; (2) used an ordinary least squares (OLS) regression model, which ignores the $0-1$ bounds on utility, instead of the twopart model; (3) calculated disutility values using discounted TTO and life expectancy amounts, using a discount rate of $3 \%$ [31]; (4) created a two-level severity measure by combining the moderate and severe groups due to the small number of children categorized into these two categories; (5) evaluated the impact of controlling for the ASD-associated conditions of epilepsy and intellectual disability in our models; and (6) did not control for parent health status in the child health utility estimation model.

\subsection{Ethics Statement}

This study was approved by the Harvard University Institutional Review Board.

\section{Results}

We received responses from $69 \%$ of parents- $72 \%$ in the ASD group $(n=145)$ and $67 \%$ in the comparison group $(n=136)$. Responders were significantly more likely to have previous internet access $(p=0.002)$ and higher education $(p=0.04)$ than non-responders, but other demographic characteristics were similar between the groups. The final sample size was 255 ( $n=135$ in the ASD group; $n=120$ in the comparison group, after applying exclusion criteria and eliminating missing or illogical responses (Online Appendix 3).

Parent respondents in the ASD group were more likely to be female, White, non-Hispanic, older, and more educated, versus the comparison group ( $p<0.05$ for all) (Table 1). Parents in the ASD group also had higher rates of anxiety, depression, migraines, and sleep disorders $(p<0.05)$ (Table 2). Income and marital status were similar between groups.

There was a higher proportion of male, older children in the ASD group $(p<0.05)$ (Table 1$)$. Allergies, anxiety, attention problems, developmental coordination problems, epilepsy, gastrointestinal problems, intellectual disability, and sleep disorder were all significantly higher among children with ASD, versus the comparison group $(p<0.05)$ (Table 2). Thirty-two percent of children with ASD had a parent-reported diagnosis of autism, $47 \%$ had a parentreported diagnosis of Asperger's syndrome, and 21\% had a parent-reported diagnosis of PDD-NOS. Using our parentreported severity measure, from 128 responses, we categorized $65 \%$ of children with ASD as mild, $17 \%$ as moderate, and $18 \%$ as severe (Table 3 ).

\subsection{Unadjusted Analyses}

Mean parent-reported child health utility values were 0.80 (95\% CI 0.75-0.86) in the comparison group and 0.56 (95\% CI 0.51-0.62) in the ASD group (Table 3). Mean parent health utility values were 0.93 (95\% CI $0.91-0.96$ ) for parents in the comparison group and 0.87 (95\% CI 0.83-0.90) for parents in the ASD group. Composite values representing child and parent health states together followed the same trend, with a higher value of 0.78 (95\% CI $0.72-0.83)$ in the comparison group versus 0.53 (95\% CI $0.48-0.58)$ in the ASD group. All mean values were calculated excluding the respondents who left the open-ended TTO questions blank (Online Appendix 4).

\subsection{Adjusted Analyses}

\subsubsection{Parent-Reported Child Health Utility}

After adjusting for respondent and child characteristics, a general diagnosis of ASD was significantly associated with a 0.12 lower (95\% CI 0.03-0.21) parent-reported child health utility (Fig. 1), a 15\% decrease from the comparison group mean. Mild and moderate levels of ASD were associated with 0.12 (95\% CI 0.03-0.20) and 0.15 (95\% CI $0.01-0.28$ ) decreases in parent-reported child health utility from the comparison group. We did not find that the most severe group had a different parent-reported child health utility than the comparison group in primary analyses, but a combined moderate/severe level of ASD was associated with a 0.14 (95\% CI 0.03-0.26) decrease in parent-reported child health utility versus the comparison group in sensitivity analyses (Online Appendix 5A). Specific diagnoses of autism and Asperger's syndrome were associated with 0.13 (95\% CI 0.02-0.25) and 0.14 (95\% CI 0.04-0.23) lower parent-reported child utility values versus the comparison group, respectively. Utility values for children diagnosed with PDD-NOS were not statistically different from the comparison group (Fig. 1). Goodness-of-fit statistics for regressions estimating the association between ASD and parent-reported child health utility, parent health utility, and parent/child composite health utility are reported in Online Appendix 6.

\subsubsection{Parent Health Utility}

On average, having a child with a general diagnosis of ASD was not significantly associated with a decrease in parent health utility in adjusted analyses (Fig. 2). However, having a child with the highest severity ASD was significantly associated with a 0.14 reduction in parent health utility $(95 \%$ CI 0.01-0.26) versus the comparison group. Having a child with less severe (mild, moderate) ASD was not statistically 
Table 1 Child and parent demographic characteristics

\begin{tabular}{|c|c|c|c|}
\hline & $\begin{array}{l}\operatorname{ASD}(\%) \\
{[n=135]}\end{array}$ & $\begin{array}{l}\text { Comparison }(\%) \\
{[n=120]}\end{array}$ & $p$ value $^{\mathrm{a}}$ \\
\hline \multicolumn{4}{|l|}{ Child demographic characteristics } \\
\hline Male $^{\mathrm{b}}$ & 81.48 & 65.55 & $<0.01$ \\
\hline \multicolumn{4}{|l|}{ Age, years } \\
\hline $3-5$ & 8.15 & 21.85 & \multirow[t]{4}{*}{0.01} \\
\hline $6-10$ & 32.59 & 34.45 & \\
\hline $11-13$ & 23.70 & 17.65 & \\
\hline $14-17$ & 35.56 & 26.05 & \\
\hline \multicolumn{4}{|l|}{ Insurance $^{c}$} \\
\hline Any: public or private & 97.04 & 93.22 & 0.15 \\
\hline \multicolumn{4}{|l|}{ Parent demographic characteristics } \\
\hline Male $^{\mathrm{d}}$ & 27.41 & 41.18 & 0.02 \\
\hline \multicolumn{4}{|l|}{ Age, years } \\
\hline $18-34$ & 18.52 & 32.77 & \multirow[t]{3}{*}{0.03} \\
\hline $35-44$ & 38.52 & 35.29 & \\
\hline $45+$ & 42.96 & 31.93 & \\
\hline \multicolumn{3}{|l|}{ Primary caregiver ${ }^{\mathrm{e}}$} & \multirow[t]{4}{*}{0.09} \\
\hline Yes & 52.63 & 40.71 & \\
\hline $\mathrm{No}^{\mathrm{f}}$ & 4.51 & 9.73 & \\
\hline $\begin{array}{l}\text { Shared evenly between respondent and another adult } \\
\text { in the household }\end{array}$ & 42.86 & 49.56 & \\
\hline \multicolumn{4}{|l|}{ Race/ethnicity } \\
\hline White, non-Hispanic & 82.96 & 68.91 & \multirow[t]{4}{*}{$<0.01$} \\
\hline Black, non-Hispanic & 4.44 & 10.08 & \\
\hline Hispanic & 6.67 & 2.52 & \\
\hline Other & 5.93 & 18.49 & \\
\hline \multicolumn{4}{|l|}{ Marital status ${ }^{\mathrm{g}}$} \\
\hline Married/living with partner & 80.00 & 81.51 & 0.76 \\
\hline \multicolumn{4}{|l|}{ Income, US\$ } \\
\hline$<25,000-50,000$ & 36.30 & 36.13 & \multirow[t]{3}{*}{0.19} \\
\hline$>50,000-100,000$ & 39.26 & 30.25 & \\
\hline$>100,000$ & 24.44 & 33.61 & \\
\hline \multicolumn{4}{|l|}{ Education } \\
\hline High school or less & 8.15 & 36.97 & \multirow[t]{3}{*}{$<0.01$} \\
\hline Some college & 38.52 & 22.69 & \\
\hline Bachelors or higher & 53.33 & 40.34 & \\
\hline \multicolumn{4}{|l|}{ US region } \\
\hline Northeast & 20.74 & 21.85 & \multirow[t]{4}{*}{0.85} \\
\hline Midwest & 23.70 & 19.33 & \\
\hline South & 30.37 & 33.61 & \\
\hline West & 25.19 & 25.21 & \\
\hline \multicolumn{4}{|l|}{ Current employment status ${ }^{\mathrm{h}}$} \\
\hline Working (paid or self) & 69.63 & 71.43 & 0.75 \\
\hline \multicolumn{4}{|l|}{ Number of children (ages 3-17 years) } \\
\hline Two or more & 67.41 & 58.82 & 0.16 \\
\hline \multicolumn{4}{|l|}{ Number of children (ages 3-17 years) with ASD } \\
\hline 1 & 93.33 & - & \multirow[t]{3}{*}{-} \\
\hline 2 & 5.19 & - & \\
\hline 3 & 1.48 & - & \\
\hline
\end{tabular}

ASD autism spectrum disorder

${ }^{\mathrm{a}} \mathrm{Chi}$-square test, differences in proportions 
Table 1 (continued)

\author{
${ }^{\mathrm{b}}$ Compared with females \\ ${ }^{c}$ Compared with those with no insurance \\ ${ }^{\mathrm{d}}$ Compared with females \\ ${ }^{\mathrm{e}}$ In the past 12 months \\ ${ }^{\mathrm{f}}$ Another adult in the household is the primary caregiver \\ ${ }^{\mathrm{g}}$ Compared with those who have been widowed/divorced/separated/never married \\ ${ }^{\mathrm{h}}$ Compared with those who answered 'not working' (on layoff, looking, retired, disabled, other)
}

associated with decreased parent health utility (Fig. 2), but in sensitivity analyses a combined moderate/severe ASD group was associated with a 0.10 (95\% CI 0.01-0.18) decrease in parent health utility versus the comparison group (Online Appendix 5B). Utility values for parents of children diagnosed with autism, Asperger's syndrome and PDD-NOS were not statistically different from the comparison group.

\subsubsection{Parent/Child Composite Health Utility}

For the parent/child composite health state (Fig. 3; Online Appendix 5C), having a child with ASD was associated with a 0.14 (95\% CI 0.02-0.22) decrease in parent/child health utility versus the comparison group. Utility losses increased with ASD severity: 0.14 for mild ASD (95\% CI 0.06-0.22), 0.15 for moderate ASD (95\% CI 0.01-0.30), and 0.34 for severe ASD (95\% CI 0.31-0.37).

\subsection{Sensitivity Analyses}

Analyses that tightened the exclusion criteria (26 additional respondents were eliminated from the ASD group) produced similar results (Online Appendix 7). For all adjusted analyses, results from the OLS regression models typically showed larger disutility estimates compared with two-part models (Online Appendix 8). Discounting TTO and life expectancy amounts by $3 \%$ produced smaller utility losses compared with the primary analysis (Online Appendix 9). Controlling for the ASD-associated conditions of epilepsy and intellectual disability in our models resulted in the same or smaller utility losses associated with ASD (Online Appendix 10). Not controlling for parent health status in the child health utility estimation models produced similar results to our primary analysis (Online Appendix 11).

\section{Discussion}

This study used parent-reported TTO estimates to derive utility values associated with the health of children with ASD and spillover effects on parents. On average, a general diagnosis of ASD (not stratified by severity) was significantly associated with a 0.11 decrease in parent-reported child health utility. Autism and Asperger's were significantly associated with a 0.13 and 0.14 decrease in parent-reported child health utility versus the comparison group, but were not significantly different from each other, which is consistent with the 2013 shift to the DSM-V diagnostic criteria, which combines these two disorders under the umbrella diagnosis of ASD. A general diagnosis of ASD was not significantly associated with spillover effects on parent health utility, but when the ASD diagnosis was stratified by severity, having a child with severe symptoms was significantly associated with a 0.14 reduction in parent health utility. Composite health utility values were significantly lower for a general ASD diagnosis and for specific types and severity levels of ASD, versus the comparison group.

Previous analyses have reported larger utility losses for children with ASD and their parents. Studies in the US and UK estimated a $0.26-0.49$ range in average child utility loss associated with ASD [17-21]. Previous parent spillover utility estimates associated with ASD in the US range from 0.09 to $0.26[21,22]$. As opposed to our method, which directly elicited utility values from parents, these studies all had parents complete a multi-attribute utility (MAU) measurement tool-the Health Utilities Index (HUI)-3 [32], EuroQol-5 Dimension (EQ-5D) [31], Short Form-6 Dimension (SF-6D) [33], or the Quality of Well-Being Scale-Self-Administered (QWB-SA) [34, 35] — to estimate parent and child utility values. These instruments facilitate indirect utility estimation, with answers weighted by adult community utility values. Differences from our estimates would be expected as smaller utility losses are often observed among patients who have experienced a condition, compared with a community sample, with adaptation to the health condition suggested as the rationale for this difference [36]. In this context, parentreported child health is closer to a patient than a community sample, and spillover estimates derived directly from parents are analogous to patient samples that have directly experienced a health condition.

For utility values included in CEAs, US guidelines recommend using an MAU instrument, such as the EQ-5D, that is completed by patients and weighted with community utility values [37]. We chose instead to use the TTO, a direct method, because of concerns that the domains in the available MAU instruments would not fully capture components of HRQOL most relevant to children with ASD, including social, communication, and behavior problems. 
Table 2 Child and parent health conditions

\section{ASD (\%) Comparison (\%) $p$ value $^{\mathrm{a}}$}

Child health conditions

ASD-associated conditions

\begin{tabular}{|c|c|c|c|}
\hline Epilepsy & 7.41 & 0.00 & $<0.01$ \\
\hline $\begin{array}{l}\text { Intellectual/cognitive dis- } \\
\text { ability }\end{array}$ & 18.52 & 0.00 & $<0.01$ \\
\hline \multicolumn{4}{|l|}{ Child health conditions } \\
\hline Allergies & 51.11 & 17.65 & $<0.01$ \\
\hline Anxiety & 34.81 & 4.20 & $<0.01$ \\
\hline Attention problems & 43.70 & 8.40 & 0.00 \\
\hline Arthritis & 1.48 & 0.84 & 0.64 \\
\hline Asthma & 9.63 & 10.08 & 0.90 \\
\hline Bipolar & 3.70 & 1.68 & 0.33 \\
\hline Cerebral palsy & 3.70 & 0.00 & 0.03 \\
\hline Depression & 6.67 & 2.52 & 0.12 \\
\hline Developmental coordination & 15.56 & 0.00 & $<0.01$ \\
\hline Diabetes & 1.48 & 0.84 & 0.64 \\
\hline Epilepsy & 7.41 & 0.00 & $<0.01$ \\
\hline Gastrointestinal diseases & 8.15 & 0.84 & 0.01 \\
\hline Hearing & 2.22 & 0.84 & 0.38 \\
\hline Heart disease & 1.48 & 0.00 & 0.18 \\
\hline Immune deficiency & 0.74 & 0.00 & 0.35 \\
\hline $\begin{array}{l}\text { Intellectual/cognitive dis- } \\
\text { ability }\end{array}$ & 18.52 & 0.00 & $<0.01$ \\
\hline Low muscle tone & 12.59 & 0.84 & $<0.01$ \\
\hline $\begin{array}{l}\text { Obsessive compulsive } \\
\text { disorder }\end{array}$ & 18.52 & 2.52 & $<0.01$ \\
\hline Pica & 5.93 & 0.00 & 0.01 \\
\hline Sleep disorder & 10.37 & 1.68 & $<0.01$ \\
\hline Tics & 5.93 & 1.68 & 0.08 \\
\hline Vision impairment & 5.19 & 0.84 & 0.05 \\
\hline \multicolumn{4}{|l|}{ Parent health conditions } \\
\hline Anxiety & 31.85 & 12.61 & $<0.01$ \\
\hline Arthritis & 11.85 & 10.08 & 0.65 \\
\hline Asthma & 12.59 & 10.92 & 0.68 \\
\hline Cancer & 1.48 & 1.68 & 0.90 \\
\hline COPD & 0.74 & 0.00 & 0.35 \\
\hline Cardiovascular disease & 0.00 & 3.36 & 0.03 \\
\hline Chronic liver disease & 0.74 & 0.00 & 0.35 \\
\hline Depression & 27.41 & 11.76 & $<0.01$ \\
\hline Diabetes & 4.44 & 7.56 & 0.29 \\
\hline Gastrointestinal & 2.96 & 0.00 & 0.06 \\
\hline High cholesterol & 13.33 & 13.45 & 0.98 \\
\hline Hypertension & 20.74 & 15.13 & 0.25 \\
\hline Migraines/severe headaches & 20.00 & 10.92 & 0.05 \\
\hline Sleep disorder & 17.04 & 5.88 & 0.01 \\
\hline
\end{tabular}

$A S D$ autism spectrum disorder, $C O P D$ chronic obstructive pulmonary disease

${ }^{\text {a }}$ Chi-square test, differences in proportions
Our estimates, which produced smaller utility losses than previous indirect methods, could indicate that these concerns about MAU instrument sensitivity were not important, or could instead reflect the expected differences in patient versus community values as described above, and we do not have the ability to disentangle these two important differences.

Incorporating family HRQOL spillover effects into CEAs has received increasing attention in the last decade, and updated guidelines for US and UK CEAs support the inclusion of these effects $[38,39]$. A previous review of the pediatric CEA literature found that incorporating family spillover effects into these analyses yields ratios that are meaningfully different from those excluding family spillover effects approximately half of the time [40]. Including these effects for CEAs of ASD therapies is especially important due to the well-known impact that ASD has on families, and our results indicate that this may be particularly true for interventions provided for the most severely affected children.

Although guidelines recommend the inclusion of family spillover effects in CEAs, there is no consensus on how best to assess these effects and incorporate them into analyses [41, 42]. In this study, we asked parents to value their own and their child's health separately and then to value their own and their child's health together in a composite health state. We found that when the child and parent utility losses are valued separately and added together, these estimates exceed the composite utility loss estimates. This may indicate that parents may have had trouble disentangling their own and their child's health in separate valuation questions $[16,43]$, although sensitivity analyses that did not control for parent comorbidities when estimating child health utility values produced similar results to our primary analyses that did. This may also suggest that the parent and child utility effects are not additive. The discrepancy observed between these two methods has been observed in previous research [13], and the potential for these differences to influence the results of CEAs highlights the need for more standard methods. Proposed frameworks being developed for estimating spillover include both additive models and multiplier effects [41, 42].

The TTO technique is one way to value health, and we have used that valuation in this study to represent a utility, but other value elicitation methods may have produced different results [44]. Specific challenges exist when the TTO technique is used in the context of valuing parent-reported child and parent health $[45,46]$. When parents are asked to trade time from their own life to improve their own health or the health of their child, they may avoid trading time because of their caregiving responsibilities [43]. We asked respondents to assume they had a 40-year life expectancy, but respondents' actual life expectancies may have influenced their TTO responses. We did not elicit values from 
Table 3 Unadjusted health utility values for child, parent, and composite

\begin{tabular}{|c|c|c|c|c|c|c|c|}
\hline \multirow[t]{2}{*}{ Group } & \multirow[t]{2}{*}{$N$} & \multicolumn{2}{|l|}{ Parent } & \multicolumn{2}{|l|}{ Child } & \multicolumn{2}{|c|}{ Composite } \\
\hline & & $\operatorname{Mean}^{\mathrm{a}}$ & $95 \% \mathrm{CI}$ & Mean $^{\mathrm{a}}$ & $95 \%$ CI & Mean $^{\mathrm{a}}$ & $95 \% \mathrm{CI}$ \\
\hline Comparison group & 120 & 0.93 & $0.91-0.96$ & 0.80 & $0.75-0.86$ & 0.78 & $0.72-0.83$ \\
\hline ASD group & 135 & 0.87 & $0.83-0.90$ & 0.56 & $0.51-0.62$ & 0.53 & $0.48-0.58$ \\
\hline \multicolumn{8}{|l|}{ Diagnostic subgroups } \\
\hline Autism & 43 & 0.87 & $0.79-0.93$ & 0.55 & $0.43-0.67$ & 0.52 & $0.41-0.64$ \\
\hline Asperger's syndrome & 64 & 0.85 & $0.80-0.89$ & 0.53 & $0.46-0.61$ & 0.49 & $0.42-0.56$ \\
\hline PDD-NOS & 29 & 0.90 & $0.83-0.95$ & 0.63 & $0.51-0.76$ & 0.65 & $0.52-0.77$ \\
\hline \multicolumn{8}{|l|}{ Composite severity subgroups } \\
\hline Severity level 1 (mild) & 83 & 0.89 & $0.86-0.93$ & 0.58 & $0.51-0.65$ & 0.55 & $0.48-0.61$ \\
\hline Severity level 2 (moderate) & 22 & 0.89 & $0.81-0.95$ & 0.56 & $0.41-0.72$ & 0.53 & $0.37-0.68$ \\
\hline Severity level 3 (severe) & 23 & 0.75 & $0.61-0.87$ & 0.49 & $0.33-0.65$ & 0.45 & $0.27-0.60$ \\
\hline
\end{tabular}

$C I$ confidence interval, $A S D$ autism spectrum disorder, $P D D$-NOS pervasive developmental disorder, not otherwise specified, TTO time trade-off

${ }^{a}$ All mean values were calculated excluding the respondents who left the open-ended TTO questions blank: 17 respondents in the comparison group and 3 in the ASD group left the child TTO blank; 14 respondents in the comparison group and 3 in the ASD group left the parent TTO question blank; 16 respondents in the comparison group and 5 in the ASD group left the composite TTO question blank (Online Appendix 4)

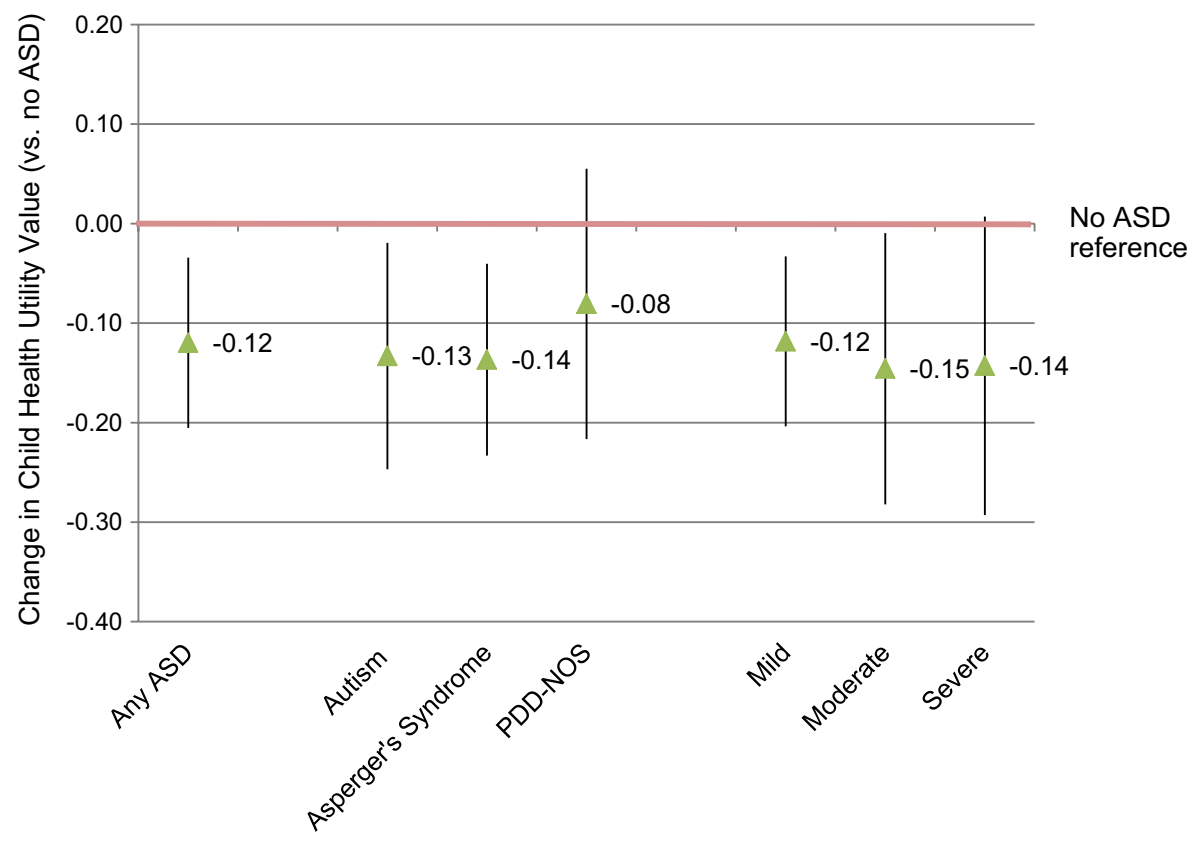

Fig. 1 Adjusted changes in child health utility values associated with having any ASD, or being in a specific diagnostic or severity subgroup of ASD, all compared with not having ASD. These regression models controlled for respondent's sex, age, race/ethnicity, income, education, marital status, child's sex, child's age, insurance status, the presence of other children in the household, and the presence of

children directly due to the cognitive demands of the TTO [43]. These concerns are particularly relevant for children with ASD, who are more likely to have intellectual, communication, and behavioral difficulties [47]. In the context of ASD, the wording that we choose for the TTO question highlights the common negative aspects of living with a child illnesses not related to the child's ASD diagnosis, and the presence of parent comorbidities. Bars around the point estimates represent bootstrapped 95\% CIs. We estimated CIs around regression coefficients using non-parametric bootstrapping procedures. ASD autism spectrum disorder, $P D D-N O S$ pervasive developmental disorder, not otherwise specified, $C I$ confidence interval

developmental disorder, for example referring to traits of ASD as 'symptoms', but does not explicitly highlight any potentially positive aspects of having a condition such as ASD, which some families celebrate [48].

There are no standard approaches for analyzing utility data due to the typically non-parametric distribution of these 


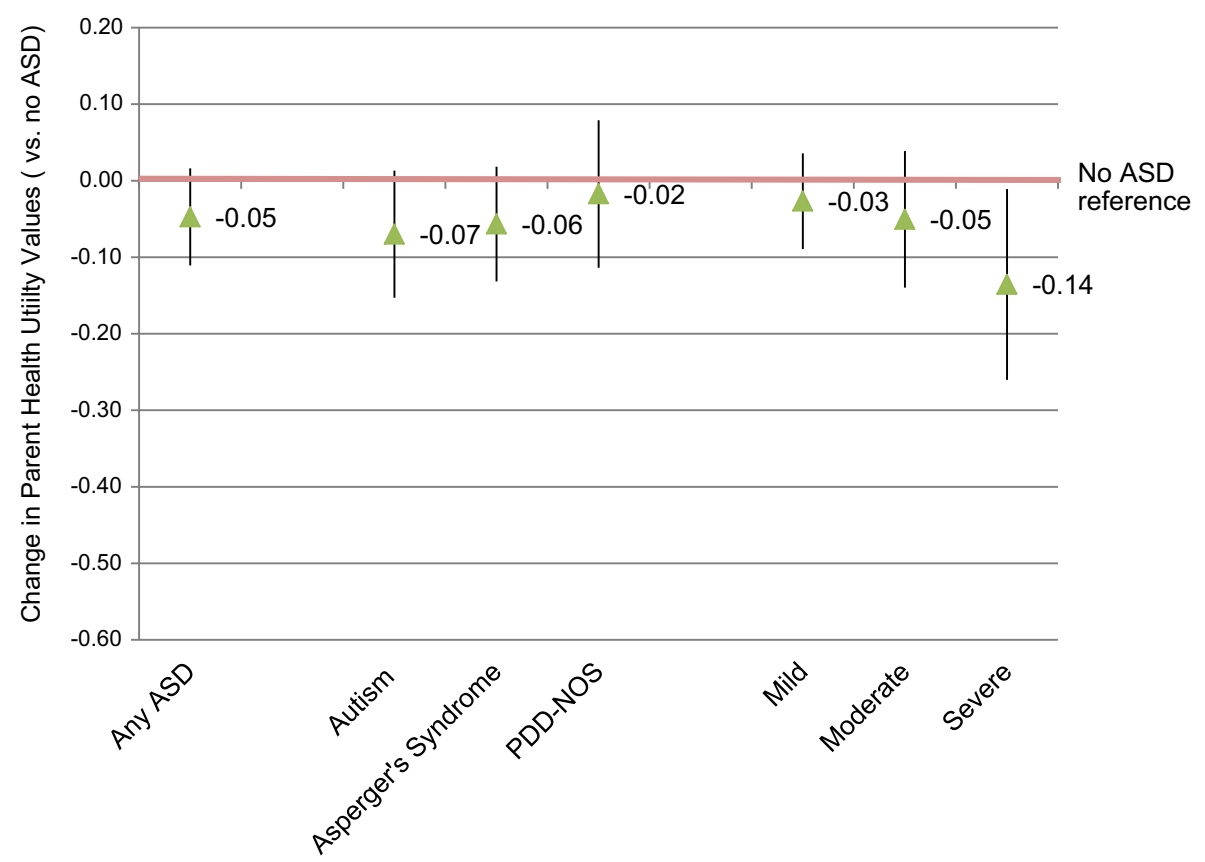

Fig. 2 Adjusted changes in parent health utility values associated with having a child with ASD, or having a child in a specific diagnostic or severity subgroup of ASD, all compared with not having a child with ASD. These regression models controlled for respondent's sex, age, race/ethnicity, income, education, marital status, child's sex, child's age, insurance status, the presence of other children in the household, and the presence of child illnesses not related to the child's ASD diagnosis. Bars around point estimates represent bootstrapped 95\% CIs. We estimated CIs around regression coefficients using non-parametric bootstrapping procedures. $A S D$ autism spectrum disorder, $P D D-N O S$ pervasive developmental disorder, not otherwise specified, $C I$ confidence interval

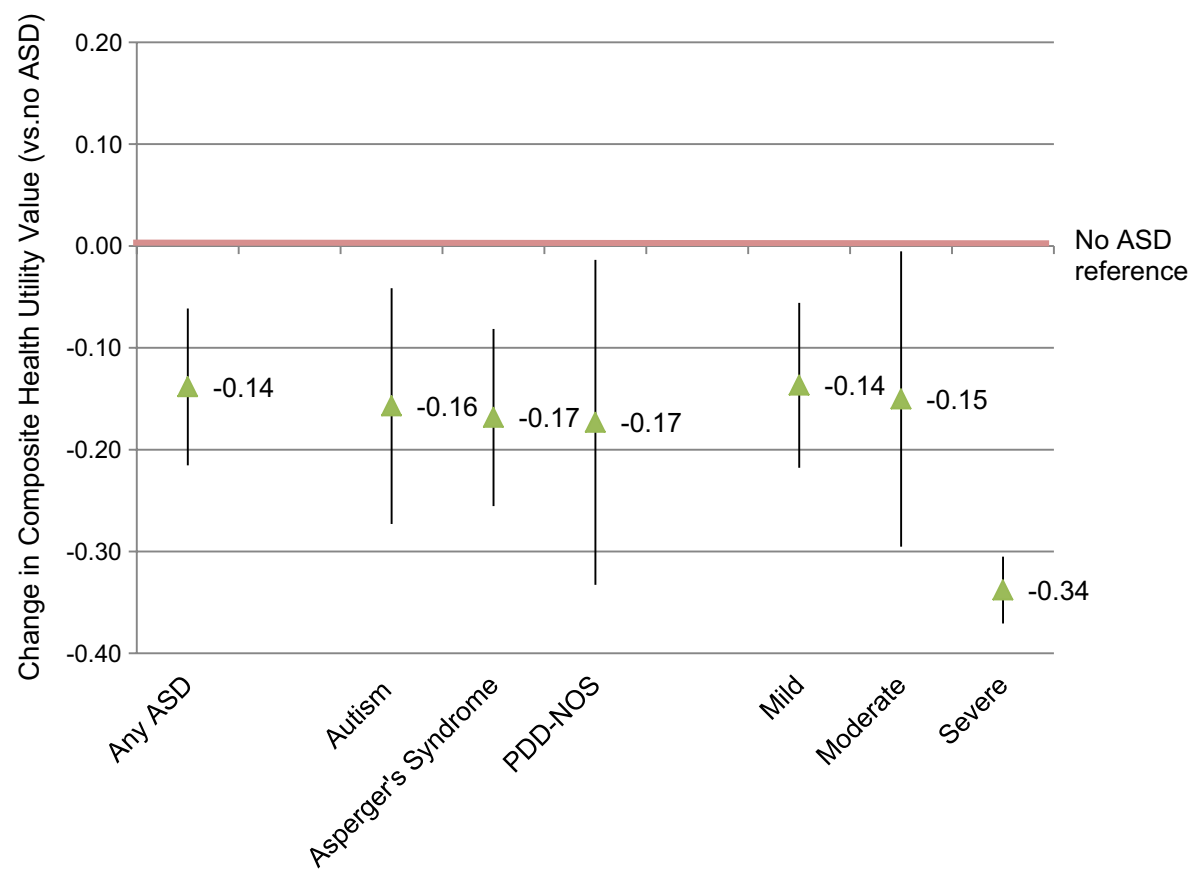

Fig. 3 Adjusted changes in composite health utility values associated with having a child with ASD, or having a child in a specific diagnostic or severity subgroup of ASD, all compared with not having a child with ASD. These regression models controlled for respondent's sex, age, race/ethnicity, income, education, marital status, child's sex, child's age, insurance status, the presence of other children in the household, and the presence of child illnesses not related to the child's ASD diagnosis. Bars around point estimates represent bootstrapped 95\% CIs. We estimated CIs around regression coefficients using non-parametric bootstrapping procedures. $A S D$ autism spectrum disorder, $P D D-N O S$ pervasive developmental disorder, not otherwise specified, $C I$ confidence interval 
data, which are bounded between 0 and 1 . We chose to use a two-part model as our utility distributions had a large proportion of utilities equal to 1 . Previous work has shown that in these circumstances, two-part models perform better than OLS [49]. As family spillover utility estimates tend to be small in magnitude [50], and sample distributions may contain a large number of perfect health utility values equal to 1 , the two-part model may be particularly well-suited to these data. In sensitivity analyses, we found that estimates of utility loss increased considerably with an OLS model.

Study limitations include a relatively small sample size, limiting generalizability and our ability to detect differences between groups. We used a parent-reported ASD diagnosis, however this has been shown to be highly reliable when verified against professional diagnostic documentation [51]. We also used a parent-reported measure of ASD severity based on levels of social communication and repetitive behavior impairments, adapted from DSM-V criteria. Clinical assessment with the Autism Diagnostic Observation Schedule (ADOS) is the gold standard for the assessment of the severity of these impairments, and misclassification with parent reporting may have occurred.

\section{Conclusions}

Our findings suggest that ASD has a significant impact on the average health utility values of children, but not of parents, however this impact is influenced by the severity of children's core social communication and repetitive behavior symptoms. Few studies have assessed the cost effectiveness of ASD interventions [52]. Although not community values, the valuations derived from these data may be useful in future CEAs of ASD interventions that demonstrate the value of interventions for ASD. As ASD interventions are aimed at symptom reduction, the utility values from this study stratified by severity level may be particularly important.

Data Availability Statement Study data are available from the authors upon reasonable request.

Acknowledgements The authors thank Joanna Emerson, MPH at the Center for the Evaluation of Value and Risk in Health, Institute for Clinical Research and Health Policy Studies, Tufts Medical Center, for research assistance.

Author Contributions Tara Lavelle designed the survey instrument, collected and analyzed all the data, and wrote and edited the manuscript. Milton Weinstein, Joseph Newhouse and Lisa Prosser gave feedback on the study design, the survey instrument development and data analyses, and edited the final manuscript. Kerim Munir and Karen Kuhlthau contributed to the survey development, gave feedback on the data analysis results, and edited the final manuscript.

\section{Compliance with Ethical Standards}

Conflicts of interest Drs. Lavelle, Weinstein, Newhouse, Prosser, and Kuhlthau report no conflicts of interest related to this study. Dr. Munir received funding from the National Institute of Mental Health Research Training Program in Mental Health and Developmental Disabilities at Boston Children's Hospital (R25MH071286), as principal investigator

Funding Funding for this study was provided by the National Institute of Mental Health (5T32MH019733-18), Harvard University's Center for American Political Studies, the National Institute of Mental Health Research Training Program in Mental Health and Developmental Disabilities at Boston Children's Hospital (R25MH071286), National Institute for Nursing Research (7-R01-NR-011880-03), and Harvard University Graduate School of Arts and Sciences.

Research Involving Human Participants This study was approved by the Harvard University Institutional Review Board.

Open Access This article is distributed under the terms of the Creative Commons Attribution-NonCommercial 4.0 International License (http://creativecommons.org/licenses/by-nc/4.0/), which permits any noncommercial use, distribution, and reproduction in any medium, provided you give appropriate credit to the original author(s) and the source, provide a link to the Creative Commons license, and indicate if changes were made.

\section{References}

1. Baio J, et al. Prevalence of autism spectrum disorder among children aged 8 years-autism and developmental disabilities monitoring network, 11 sites, United States, 2014. Morb Mortal Wkly Rep. 2018;67(6):1-23.

2. Baxter AJ, et al. The epidemiology and global burden of autism spectrum disorders. Psychol Med. 2015;45(3):601-13.

3. Weinstein MC, Torrance G, McGuire A. QALYs: the basics. Value Health. 2009;12(Suppl 1):S5-9.

4. Gold MR. Cost-effectiveness in health and medicine. New York: Oxford University Press; 1996. p. 425.

5. Lin P-J, D'Cruz B, Leech A, Neumann PJ, Aigbogun MS, Oberdhan D, Lavelle T. Family and caregiver spillover effects in alzheimer's disease cost-effectiveness analyses. Alzheimer's Dementia. 2017;13:P1565-6. https://doi.org/10.1016/j.jalz.2017.07.720.

6. Leech et al. Family and caregiver spillover effects in cancer costeffectiveness analyses. In: Society for medical decision making 39th annual North American meeting. 22-25 Oct 2017: Pittsburg, PA

7. American Psychiatric Association and DSM-5 Task Force. Diagnostic and statistical manual of mental disorders. 5th ed. Washington, DC: American Psychiatric Association; 2013.

8. Karst JS, Van Hecke AV. Parent and family impact of autism spectrum disorders: a review and proposed model for intervention evaluation. Clin Child Fam Psychol Rev. 2012;15(3):247-77.

9. Hastings RP, Johnson E. Stress in UK families conducting intensive home-based behavioral intervention for their young child with autism. J Autism Dev Disord. 2001;31(3):327-36.

10. Montes G, Halterman JS. Psychological functioning and coping among mothers of children with autism: a population-based study. Pediatrics. 2007;119(5):e1040-6.

11. Schieve LA, et al. The relationship between autism and parenting stress. Pediatrics. 2007;119(Suppl 1):S114-21. 
12. Basu A, Meltzer D. Implications of spillover effects within the family for medical cost-effectiveness analysis. J Health Econ. 2005;24(4):751-73.

13. Prosser LA, et al. Measuring family HRQoL spillover effects using direct health utility assessment. Med Decis Making. 2015;35(1):81-93.

14. Al-Janabi H, Flynn TN, Coast J. QALYs and Carers. PharmacoEconomics. 2011;29(12):1015-23.

15. Prosser LA. Current challenges and future research in measuring preferences for pediatric health outcomes. J Pediatr. 2009;155(1):7-9.

16. Ungar WJ. Challenges in health state valuation in paediatric economic evaluation: are QALYs contraindicated? Pharmacoeconomics. 2011;29(8):641-52.

17. Petrou S, et al. Economic costs and preference-based healthrelated quality of life outcomes associated with childhood psychiatric disorders. Br J Psychiatry. 2010;197(5):395-404.

18. Petrou S, Kupek E. Estimating preference-based health utilities index mark 3 utility scores for childhood conditions in England and Scotland. Med Decis Making. 2009;29(3):291-303.

19. Payakachat $\mathrm{N}$, et al. Autism spectrum disorders: a review of measures for clinical, health services and cost-effectiveness applications. Expert Rev Pharmacoecon Outcomes Res. 2012;12(4):485-503.

20. Tilford JM, et al. Preference-based health-related quality of life outcomes in children with autism spectrum disorders: a comparison of generic instruments. PharmacoEconomics. 2012;30(8):661-79.

21. Tilford JM, et al. Treatment for sleep problems in children with autism and caregiver spillover effects. J Autism Dev Disord. 2015;45:3613-23.

22. Khanna R, Jariwala K, Bentley JP. Health utility assessment using EQ-5D among caregivers of children with autism. Value Health. 2013;16(5):778-88.

23. GfK. Consumer panels [cited 19 Dec 2018]. https://www.gfk.com/ en-us/solutions/consumer-panel/. Accessed 19 Dec 2018.

24. Werner E, et al. Variation in early developmental course in autism and its relation with behavioral outcome at 3-4 years of age. J Autism Dev Disord. 2005;35(3):337-50.

25. Torrance GW. Utility approach to measuring health-related quality of life. J Chronic Dis. 1987;40(6):593-603.

26. Wittenberg E, Prosser LA. Ordering errors, objections and invariance in utility survey responses. Appl Health Econ Health Policy. 2011;9(4):225-41.

27. Efron B. Better bootstrap confidence-intervals. J Am Stat Assoc. 1987;82(397):171-85.

28. Wittenberg E, et al. The effect of age, race and gender on preference scores for hypothetical health states. Qual Life Res. 2006;15(4):645-53.

29. Shaw JW, Johnson JA, Coons SJ. US valuation of the EQ-5D health states: development and testing of the D1 valuation model. Med Care. 2005;43(3):203-20.

30. Dolan P, Roberts J. To what extent can we explain time trade-off values from other information about respondents? Soc Sci Med. 2002;54(6):919-29.

31. Dolan P, Jones-Lee M. The time trade-off: a note on the effect of lifetime reallocation of consumption and discounting. J Health Econ. 1997;16(6):731-9.

32. Horsman $\mathrm{J}$, et al. The Health Utilities Index (HUI $\left.{ }^{\circledR}\right)$ : concepts, measurement properties and applications. Health Qual Life Outcomes. 2003;1:54-54.
33. Craig BM, et al. US valuation of the SF-6D. Med Decis Making. 2013;33(6):793-803.

34. Kaplan R, Anderson J. A general health policy model: update and applications. Health Serv Res. 1988;23(2):03-35.

35. Kaplan RM, et al. The quality of well-being scale. Applications in AIDS, cystic fibrosis, and arthritis. Med Care. 1989;27(3 Suppl):S27-43.

36. Ubel PA, Loewenstein G, Jepson C. Whose quality of life? A commentary exploring discrepancies between health state evaluations of patients and the general public. Qual Life Res. 2003;12(6):599-607.

37. Sanders GD, et al. Recommendations for conduct, methodological practices, and reporting of cost-effectiveness analyses: second panel on cost-effectiveness in health and medicine. JAMA. 2016;316(10):1093-103.

38. Neumann PJ, Sanders GD. Cost-effectiveness analysis 2.0. N Engl J Med. 2017;376(3):203-205.

39. National Institute for Health and Care Excellence (NICE). Guide to the methods of technology appraisal. London: National Institute for Health and Care Excellence; 2013.

40. Lavelle TA, et al. Family spillover effects in pediatric cost-utility analyses. Appl Health Econ Health Policy. Epub 22 Oct 2018. https://doi.org/10.1007/s40258-018-0436-0.

41. Hoefman RJ, van Exel J, Brouwer WB. Measuring the impact of caregiving on informal carers: a construct validation study of the CarerQol instrument. Health Qual Life Outcomes. 2013;11:173.

42. Al-Janabi $\mathrm{H}$, et al. A framework for including family health spillovers in economic evaluation. Med Decis Making. 2016;36(2):176-86.

43. Prosser LA, Hammitt JK, Keren R. Measuring health preferences for use in cost-utility and cost-benefit analyses of interventions in children: theoretical and methodological considerations. Pharmacoeconomics. 2007;25(9):713-26.

44. Arnold D, et al. Comparison of direct and indirect methods of estimating health state utilities for resource allocation: review and empirical analysis. BMJ. 2009;339:b2688.

45. Johannesson M, Pliskin J, Weinstein M. A note on QALYs, time tradeoff, and discounting. Med Decis Making. 1994;14(2):188-93.

46. Prosser LA, et al. Preferences and willingness to pay for health states prevented by pneumococcal conjugate vaccine. Pediatrics. 2004;113(2):283-90.

47. Gnanavel S, Robert RS. Diagnostic and statistical manual of mental disorders, fifth edition, and the impact of events scale-revised. Chest. 2013;144(6):1974.

48. Hoefman R, et al. Caring for a child with autism spectrum disorder and parents' quality of life: application of the CarerQol. J Autism Dev Disord. 2014;44(8):1933-45.

49. Basu A, Manca A. Regression estimators for generic healthrelated quality of life and quality-adjusted life years. Med Decis Making. 2012;32(1):56-69.

50. Wittenberg E, Prosser LA. Disutility of illness for caregivers and families: a systematic review of the literature. Pharmacoeconomics. 2013;31(6):489-500.

51. Daniels AM, et al. Verification of parent-report of child autism spectrum disorder diagnosis to a web-based autism registry. J Autism Dev Disord. 2012;42(2):257-65.

52. Lamsal R, Zwicker JD. Economic evaluation of interventions for children with neurodevelopmental disorders: opportunities and challenges. Appl Health Econ Health Policy. 2017;15(6):763-72. 